\title{
XXVIII. A new and general solution of cubic equations
}

\author{
John T. Graves Esq. M.A.
}

To cite this article: John T. Graves Esq. M.A. (1838) XXVIII. A new and general solution of cubic equations, Philosophical Magazine Series 3, 13:81, 217-219, DOI: 10.1080/14786443808649556

To link to this article: http://dx.doi.org/10.1080/14786443808649556

曲 Published online: 01 Jun 2009.

Submit your article to this journal ๘

Џ Article views: 3

Q View related articles ¿ 
their metallic surfaces) proceeds as a natural consequence without the aid of any hypotheses. The various action of the insulated pile is still enigmatic, but might possibly depend on this, that with a bad conducting fluid the amount of conduction of the pile becomes greater, since then the electricity probably penetrates even between fluid and metallic surface, which might be less the case if the conducting power of the fluid approached more to that of the metal; a subject moreover on which special experiments are still desirable.

[To be continued.]

XXVIII. A Nere and General Solution of Cubic Equations. By John T. Gkaves, of the Inner Temple, Esq., M.A.*

I $\mathrm{N}$ the ordinary books of algebra, (so far, at least, as my limited acquaintance with them extends,) where cubic equations are discussed, the cases of real coefficients only are considered, and different methods of solution are given in order to effect the separation between the constituents $\dagger$ of the roots in different cases. I have obtained a symmetrical solution of the equation

$$
x^{3}+(x+\sqrt{-1} \lambda) x+\mu+\sqrt{-1} \nu=0
$$

which presents the constituents of $x$ in an explicit form in all cases. This is all that is wanted, for the solution of a cubic equation of the general form.

$$
\begin{gathered}
\left(\alpha_{1}+\sqrt{-1} \beta_{1}\right) y^{3}+\left(\alpha_{2}+\sqrt{-1} \beta_{2}\right) y^{8}+\left(\alpha_{3}+\sqrt{-1} \beta_{3}\right) y \\
+\alpha_{4}+\sqrt{-1} \beta_{4}=0
\end{gathered}
$$

may easily be made to depend on the solution of a cubic equation of the form (1.); and, from the nature of the relation between the transformed equation (1.) and the original equation (2.), the constituents $(x, \lambda, \mu, \nu)$ of the transformed coefficients $(x+\sqrt{-1} \lambda, \mu+\sqrt{-1} \nu)$ are easily determinable, supposing the constituents of the original coefficients to be explicitly given: and if the constituents of $x$ be determinable, those of $y$ can easily be determined.

The limits of this Magazine do not permit an exposition of my process, which I intend hereafter to communicate at length through some more appropriate medium. It consists in an analysis of the following formula for $x$.

$x=-\sqrt{-1} \frac{2}{\sqrt{3}} p^{\frac{1}{2}} \cos \left\{\frac{1}{3} \cos ^{-1}\left(\sqrt{-1} \frac{3 \sqrt{3}}{2} \frac{q}{p p_{1}^{\frac{1}{2}}}\right)\right\}$

where $p=x+\sqrt{-1} \lambda$ and $q=\mu+\sqrt{-1} \nu$, and where the

* Communicated by the Author.

$\uparrow$ I call $\alpha$ and $\beta$ the "constituents" of the expression $\alpha+\sqrt{-1} \beta$. 
ambiguous $p^{\frac{1}{2}}$ may have either of its two values, provided it retain at any one time the same meaning in both places of its occurrence in formula (3.).

My result is presented in the following formulæ, in which $\sqrt{ }$ and $\sqrt[3]{ }$ denote real roots not negative: $\cos _{0}{ }^{-1}$ denotes the smallest $\cos ^{-1}$ not negative: and $i$ denotes any term of the arithmetical series $-2,-1,0,1,2$, reckoned from 0 indefinitely backward and forward.

$$
\begin{aligned}
& \text { Let } \left.\begin{array}{rl}
\mathbf{Q}_{1} & =\frac{1}{\sqrt{2}} \sqrt{\sqrt{x^{2}+\lambda^{2}}+x} \\
\mathbf{Q}_{2} & =\frac{1}{\sqrt{2}} \sqrt{\sqrt{x^{2}+\lambda^{2}-x}}
\end{array}\right\} \\
& \left.\left.\begin{array}{ll}
x_{1}=2^{2}\left(x^{3}-3 x \lambda^{2}\right) \\
\lambda_{1}=2^{2}\left(3 x^{2} \lambda-\lambda^{3}\right)
\end{array}\right\} \quad \text { (5.) } \quad \begin{array}{l}
\mu_{1}=3^{3}\left(\mu^{2}-\nu^{2}\right) \\
\nu_{1}=3^{3} \cdot 2 \mu \nu
\end{array}\right\}
\end{aligned}
$$

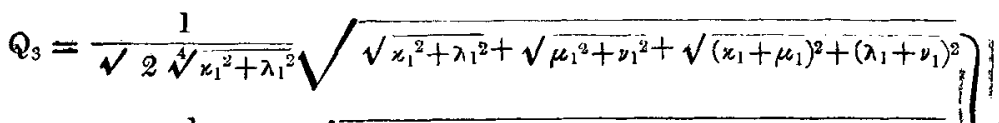

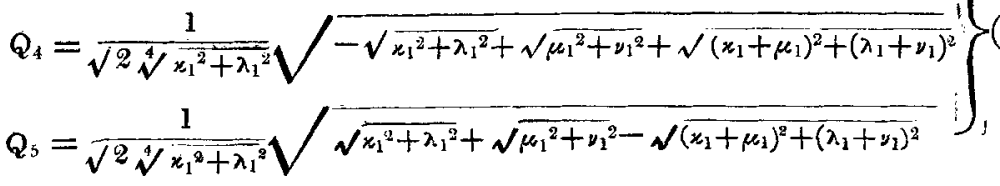

$$
\begin{aligned}
& \left.\begin{array}{l}
\mathrm{R}_{\mathbf{1}}=\frac{1}{\sqrt{3}}\left(\sqrt[3]{Q_{3}+Q_{4}}+\sqrt[3]{Q_{3}-Q_{4}}\right) \cos \left\{\frac{1}{3}\left(2 i \pi+\cos _{0}-1 Q_{5}\right)\right\} \\
\mathrm{R}_{2}=\frac{1}{\sqrt{3}}\left(\sqrt[3]{Q_{3}+Q_{4}}-\sqrt[3]{Q_{3}-Q_{4}}\right) \sin \left\{\frac{1}{3}\left(2 i \pi+\cos _{0}-1 Q_{5}\right)\right\}
\end{array}\right\} \\
& s_{1}=2 \mathbf{Q}_{1}^{2}(x \mu+\lambda \nu)+\lambda(x \nu-\lambda \mu) \\
& s_{2}=2 \mathrm{Q}_{2}{ }^{2}(x \mu+\lambda \nu)-\lambda(x \nu-\lambda \mu) \\
& s_{3}=2 \mathrm{Q}_{1}^{2}\left(x^{\nu}-\lambda \mu\right)-\lambda(x \mu+\lambda \nu) \\
& \left.s_{4}=2 \mathrm{Q}_{2}{ }^{2}(x \nu-\lambda \mu)+\lambda(x \mu+\lambda \nu)\right] \\
& x=v_{1}+\sqrt{-1} v_{2} \\
& \left.\begin{array}{l}
v_{1}=\frac{s_{1}}{\sqrt{s_{1}^{2}}} Q_{1} R_{2}+\frac{s_{2}}{\sqrt{s_{2}{ }^{2}}} Q_{2} R_{1} \\
v_{2}=\frac{s_{3}}{\sqrt{s_{3}{ }^{2}}} Q_{1} R_{1}+\frac{s_{4}}{\sqrt{s_{4}{ }^{3}}} Q_{2} R_{2}
\end{array}\right\}
\end{aligned}
$$

From the preceding formulæ it would be easy, did the size of the page permit, to write out at full length a solution for $x$ in immediate terms of $x, \lambda, \mu, \%$ The relations inter se of the mediate functions employed are very remarkable. It will be seen that $s_{1}, s_{2}, s_{3}$, and $s_{4}$ are wanted merely as sign-indicators. The critical cases where a sign-indicator becomes $=0$, are im- 
portant. It is also interesting to consider the conditions that are necessary in order that a root should be wholly real or wholly imaginary; and to observe the curious manner in which, when $\lambda$ and $\nu$ are both $=0$, the solution here given identifies itself respectively, according to certain relations between $x$ and $\mu$, with the ordinary algebraical or trigonometrical solution.

\section{Note on the Constitution of Salts. By Professor T. Graham *.}

THE author may perhaps be excused in drawing the attention of chemists to a distinction in saline combinations, which is at present too often overlooked, and confusion thereby occasioned. The orders of monobasic, bibasic, and tribasic salts, of which the phosphates proved types, have lately been greatly enlarged by the discoveries of Liebig and Dumas respecting vegetable acids, and the distinctive characters of these orders are well understood. The best proof of an acid's being bibasic or tribasic, is its combining at once with two bases which are isomorphous, or belong to the same natural family, as phosphoric acid does with soda and ammonia in microcosmic salt, and tartaric acid with potash and soda in Rochelle salt. Water and magnesia, water and barytes, water and oxide of lead, are also constantly associated as bases in bibasic and tribasic salts, but never in true double salts, or combinations of two or more salts with each other, with which salts of the preceding orders are apt to be confounded.

But it is too generally supposed, that a metallic oxide cannot exist in a saline combination, except in the capacity of base, although in most of those bodies which are at present termed subsalts, the whole or a portion of the metallic oxide is certainly not basic, but is attached to a really neutral salt in a capacity similar to that of constitutional water, or water of crystallization. Oxide of copper, oxide of lead, barytes, and the other metallic oxides included in or related to the magnesian family, appear to rival water (which is a member of the same family), in the frequency with which they discharge this function in the constitution of saline compounds, particularly of those belonging to the organic kingdom. Thus the neutral organic principle orcine combines with five atoms of oxide of lead, according to Dumas, which replace five atoms of water which orcine otherwise possesses. But it should be brought prominently into view, that neither the water nor the oxide of

- Read before the Chemical Section of the British Association, at the late meeting at Newcastle, Aug. 22, 1838; and now communicated by the Author. 